\title{
Guest editorial: Privacy-aware multimedia surveillance systems
}

\author{
Pradeep K. Atrey · Sabu Emmanuel • \\ Sharad Mehrotra $\cdot$ Mohan S. Kankanhalli
}

Published online: 23 September 2011

(C) Springer-Verlag 2011

Due to the significant increase in various security threats, safety has become a primary concern for our society. As a result, most of the public places such as airports, train stations, banks, shopping malls, subways and streets are nowadays equipped with state-of-the-art multimedia surveillance systems. These systems are meant to process sensory data to automatically detect suspicious or unusual behavior of people and alert security personnel so that preventive actions can be taken. Although such surveillance infrastructure has proved to be very useful from a security perspective; there has been apprehension among people in regard to their privacy safeguards. Citizens have increasingly begun to object to being watched electronically. Hence, there is a need for preserving privacy of people yet providing them a sense of security through effective surveillance. It is worth mentioning that although there has been a significant progress in the field of surveillance research, the issues related to people's privacy

\footnotetext{
P. K. Atrey $(\bowtie)$

Department of Applied Computer Science,

University of Winnipeg, Winnipeg, Canada

e-mail: p.atrey@uwinnipeg.ca

S. Emmanuel

School of Computer Engineering, Nanyang Technological

University, Singapore, Singapore

e-mail: asemmanuel@ntu.edu.sg

S. Mehrotra

Department of Computer Science, University of California, Irvine, USA

e-mail: sharad@ics.uci.edu

M. S. Kankanhalli

School of Computing, National University of Singapore, Singapore, Singapore

e-mail: mohan@comp.nus.edu.sg
}

have often been overlooked in the past and have only begun to attract the attention of researchers very recently. The goal of this special issue is to bring forth the recent advances in the privacy research for multimedia surveillance.

We received nine submissions from an open call for papers that address different aspects of privacy-aware multimedia surveillance systems. Although many submissions were of good quality, guest editorial committee recommended to accept only five top quality papers after a careful and highly competitive review process. These papers cover diverse issues, including privacy protection using Chaos-cryptography-based data scrambling and Markov chain algorithms, privacy filters in live surveillance video, preserving privacy in mobile video surveillance, and community-based user-specific and location-aware privacy awareness.

The first paper of this special issue "User centric privacy protection in video surveillance" by Thomas Winkler and Bernhard Rinner presents a concept for user-centric privacy awareness in video surveillance. The proposed system follows a community-based approach and empowers monitored persons to actively participate in registering cameras using their conventional smart phones. The collected information is used to warn users of violations of their personal privacy policy. Moreover, the proposed system is scalable in terms of different levels of privacy.

The second paper "A general framework for managing and processing live video data with privacy protection" by Alexander J. Aved and Kien A. Hua describes the live video database model with an intrinsic privacy model that provides a level of privacy protection not previously available for real-time streaming video data. The authors present the query language LVSQL, the system architecture, the object recognition and cross-camera tracking 
technique, and privacy filters. Privacy filters can be specified at different levels, i.e. users, cameras, and query.

The third paper "Chaos-cryptography based privacy preservation technique for video surveillance" by Sk. Md. Mizanur Rahman, M. Anwar Hossain, Hussein Mouftah, Abdulmotaleb El Saddik and Eiji Okamoto proposes a Chaos-cryptography-based data scrambling method to hide the privacy-sensitive regions of interest (ROI) in a surveillance video. The chaotic system makes use of Henon map as the logistic function, which generates the chaotic sequences. The chaotic sequences are in turn used to generate the bit sequences that controls the ROI scrambling. The proposed approach is computationally efficient and, hence, it can be applied for real-time video surveillance tasks in preserving privacy sensitive information.

The fourth paper "Intended human object detection for automatically protecting privacy in mobile video surveillance" by Yuta Nakashima, Noboru Babaguchi and Jianping Fan introduces a new concept called intended human objects that are defined as human objects essential for capture intentions, and develops a new method called intended human object detection that automatically detects the intended human objects in mobile surveillance videos. Through the process of intended human object detection, authors develop a system for automatically obscuring privacy sensitive regions.

The fifth and last paper of this issue "Privacy enabled video surveillance using a two state Markov tracking algorithm" by Peng Zhang, Tony Thomas and Sabu Emmanuel presents a novel, on-demand selectively revocable, privacy preserving mechanism for pedestrians in a surveillance video. In the proposed scheme, a surveillance video can be tuned to be viewed with complete privacy or by revoking the privacy of any subset of pedestrians while ensuring complete privacy to the remaining pedestrians. Authors achieve this by tracking the pedestrians using a novel Markov chain algorithm with two hidden states, detecting the head contour of the tracked pedestrians and obscuring their faces using an encryption mechanism. The detected pedestrian face/head is obscured by encrypting with a unique key derived from a master key for the privacy preservation purpose.

The guest editorial team thanks all the authors for submitting their quality work to this special issue, and to the numerous reviewers for their hard work and expert comments that proved highly useful for the success of this special issue. Our special thanks go to Prof. Thomas Plagemann, Editor-in-Chief for his invaluable guidance all through the process of this special issue.

\section{Author Biographies}

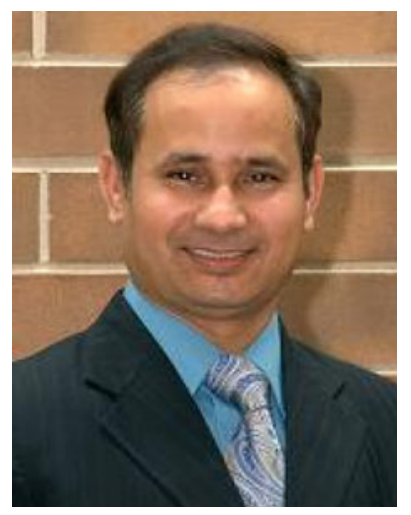

Pradeep K. Atrey is an Assistant Professor and a Senator at the University of Winnipeg, Canada. He received his Ph.D. in Computer Science from National University of Singapore, M.S. in Software Systems and B.Tech. in Computer Science and Engineering from India. He was a Postdoctoral Researcher at the Multimedia Communications Research Laboratory, University of Ottawa, Canada. His current research interests are in the area of Multimedia Computing with a focus on Multimedia Surveillance and Privacy, Image/Video Security, and Web. He has authored/co-authored over 55 research articles at reputed ACM, IEEE, and Springer journals and conferences. Dr. Atrey is on the editorial board of ETRI Journal and Journal of Convergence (Web and Multimedia). He is actively involved in his research community and he has been associated with over 20 international conferences in various roles such as General Chair, Program Chair, Publicity Chair, Web Chair, and TPC Member. Dr. Atrey was recipient of the ETRI Journal Best Reviewer Award (2009) and the University of Winnipeg Merit Award for Exceptional Performance (2010). He was also recognized as "ICME 2011-Quality Reviewer".

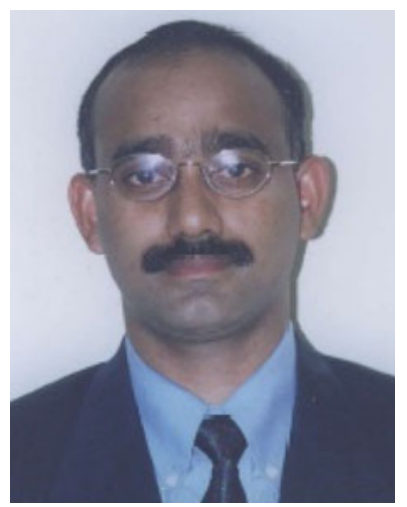

Sabu Emmanuel is currently an Assistant Professor in the School of Computer Engineering, NTU, Singapore. He received his BE (Electronics and Communication Engineering) from Regional Engineering College, Durgapur (1988), ME (Electrical Communication Engineering) from the Indian Institute of Science (IISc.), Bangalore (1998), and Ph.D. (Computer Science) from the National University of Singapore (NUS), Singapore (2002). He began his career as a research and development engineer and then moved on to the academic profession. $\mathrm{He}$ has taught engineering students of Mangalore University, National University of Singapore and Nanyang Technological University. His current research interests are in multimedia and software security, and surveillance video processing. He has served as guest editor for special issue of several journals and also reviewer for several journals such as Springer multimedia systems, IEEE transactions on circuits and systems for video technology, IEEE transactions on multimedia, IEEE transactions on information forensics and security. He has also served as technical program committee member for several conferences. 


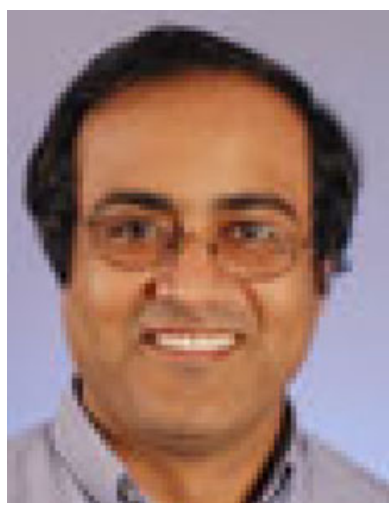

Sharad Mehrotra is a Professor in the School of Information and Computer Science at University of California, Irvine (UCI) and Director of the Center for Emergency Response Technologies (CERT) at UCI. Mehrotra's research expertise is in data management and distributed systems areas in which he has made many pioneering contributions. Two such contributions include the concept of "database as a service" and "use of information retrieval techniques, particularly relevance feedback, in multimedia search". Mehrotra is a recipient of numerous best paper nominations and awards including SIGMOD Best Paper award (2001), Best of VLDB submissions (1994), and best paper award in DASFAA (2004). Mehrotra's current research focuses on building sentient spaces using multimodal sensors, data privacy, and data quality. Mehrotra's recent research, particularly, in the context of CERT has focused on situational awareness from multimodal input including conversationalspeech data. Many of his research contributions have been incorporated into software artifacts which are now in use at various first responder partner sites. He holds a patent on privacy protection of data collection in pervasive environments.

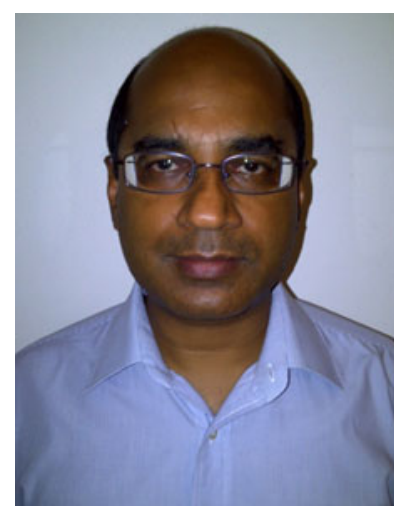

Mohan S. Kankanhalli is a Professor at the Department of Computer Science of the National University of Singapore. He is also the Associate Provost for Graduate Education at NUS. Before that, he was the Vice-Dean for Academic Affairs and Graduate Studies at the NUS School of Computing during 2008-2010 and Vice-Dean for Research during 2001-2007. Mohan obtained his BTech (Eletrical Eng.) from the Indian Institute of Technology, Kharagpur, in 1986 and his MS and Ph.D. (Computer \$ Systems Eng.) from the Rensselaer Polytechnic Institute in 1998 and 1990, respectively. He was a researcher at the Institute of Systems Science at NUS during 1990-1997. He then became a faculty member at the Department of Electrical Engineering of the Indian Institute of Science, Bangalore during 1997-1998 after which he joined NUS again. He visited the University of California at Berkeley during Jan-Jun 2004. He is actively involved in the Multimedia Systems community and he is currently the Director of Conferences for ACM SIG Multimedia. He is on the editorial boards of several journals including the ACM Transactions on Multimedia Computing, Communications, and Applications, Springer Multimedia Systems Journal, Pattern Recognition Journal and Multimedia Tools \& Applications. His current research interests are in Multimedia Systems (content processing, retrieval) and Multimedia Security (surveillance, digital rights management and privacy). 